\title{
Spontaneous chiral-symmetry breaking of lattice QCD with massless dynamical quarks *
}

\author{
Xiang-Qian Luo \\ Department of Physics, Zhongshan (Sun Yat-Sen) University, Guangzhou 510275, China ${ }^{\dagger}$
}

October 13, 2018

\begin{abstract}
One of the most challenging issues in QCD is the investigation of spontaneous chiralsymmetry breaking, which is characterized by the non-vanishing chiral condensate when the bare fermion mass is zero. In standard methods, one has to perform expensive lattice simulations at multiple bare quark masses, and employ some modeled function to extrapolate the data to the chiral limit. This paper applies the probability distribution function method to computing the chiral condensate in lattice QCD with massless dynamical quarks, without any ambiguous mass extrapolation. The results for staggered quarks indicates that the method might be a more efficient alternative for investigating the spontaneous chiral-symmetry breaking in lattice QCD.
\end{abstract}

\section{INTRODUCTION}

Chiral-symmetry breaking is an important feature of quantum field theory with fermions. In QCD with massless quarks at low temperature or density, the system is confined and chiral symmetry is spontaneously broken. At sufficiently high temperature or density, there is a chiral phase transition from the confinement phase to the quark-gluon plasma phase or color-superconducting phase, where chiral symmetry is restored. There have been many phenomenological or QCDinspired model studies of spontaneous chiral-symmetry breaking and chiral phase transitions, but lattice gauge theory (LGT)[1] should give the most reliable information from the first principles. These issues $[2,3,4,5,6,7,8,9,10]$ have been extensively investigated on the lattice for a long time, but there are still many unsolved problems.

The main question we would address here is how to get quantitative information on spontaneous chiral-symmetry breaking from lattice simulations with dynamical quarks. When the bare fermion mass $m$ is zero, the action is invariant under the global chiral transformation of the fermion field $\psi \rightarrow \exp \left(i \boldsymbol{\alpha} \cdot \boldsymbol{\tau} \gamma_{5}\right) \psi$, with $\boldsymbol{\tau}$ the generator of the chiral symmetry group, but the

\footnotetext{
${ }^{*}$ Supported by the Key Project of National Science Foundation (10235040), Key Project of National Ministry of Eduction (105135), Project of the Chinese Academy of Sciences (KJCX2-SW-N10) and Guangdong Ministry of Education.

${ }^{\dagger}$ Email: stslxq@zsu.edu.cn
} 
bilinear operator $\bar{\psi} \psi$ changes. A non-vanishing vacuum expectation value $\left.\langle\bar{\psi} \psi\rangle\right|_{m=0}$ is the signal for spontaneous chiral-symmetry breaking, which is also associated with dynamical generation of fermion mass.

Suppose the quarks are degenerate. The chiral condensate per flavor is

$$
\langle\bar{\psi} \psi\rangle=\left\langle\operatorname{Tr} \Delta^{-1}\right\rangle /\left(N_{c} V\right),
$$

where $N_{c}=3$ is the number of colors, $V$ is the number of lattice sites and $\Delta$ is the fermonic matrix. The trace is taken in the color, spin and position space. The expectation value " $<\ldots>$ " is computed with the integration measure associated with the partition function

$$
Z=\int[d \bar{\psi}][d \psi][d U] \exp (-S)=\int[d U] \exp \left(-S_{g}+\ln \operatorname{det} \Delta\right),
$$

where $U$ is the gauge link variable, $S_{g}$ is the gluonic action, and $\Delta=m+i \Lambda$ is the fermionic matrix.

A popular way to calculate the chiral condensate is to use the matrix inversion technique. Another popular way is to use

$$
\langle\bar{\psi} \psi\rangle=\frac{1}{N_{c} V}\left\langle\sum_{j=1}^{N c V / 2} \frac{2 m}{\lambda_{j}^{2}+m^{2}}\right\rangle,
$$

with $\lambda_{j}$ the $j$-th positive eigenvalue of $\Lambda$. The disadvantage is that it requires the calculation of all eigenvalues of the Dirac operator. When the lattice volume is large, the computational task is huge and not so feasible. It is also very expensive to generate configurations with dynamical quarks at multiple fermion masses. To get the chiral condensate in the chiral $\operatorname{limit}_{\lim } \lim _{V \rightarrow \infty}\langle\bar{\psi} \psi\rangle$, it requires a $m$ extrapolation, since $\left\langle\bar{\psi} \psi>\left.\right|_{m=0}=0\right.$ on a finite lattice. Such an extrapolation of $\langle\bar{\psi} \psi\rangle$ to the massless limit uses some modeled fitting functions (e.g., linear function, plus quadratic or logarithmic corrections). Unfortunately, such a process might not be well justified, and sometimes it gives arbitrary results, in particular when the system is at a phase transition point.

In Ref.[11], an alternative, named the probability distribution function (p.d.f.) method of the chiral condensate was proposed to investigate spontaneous chiral-symmetry breaking in LGT with fermions. The p.d.f. method has been tested in the Schwinger model[11], and applied to the investigation of the spontaneous $\mathrm{P}$ and CT symmetry breaking[12], theta-vacuum like systems[13] and the phase transition of SU(2) LGT at finite density[14], as well as SU(3) LGT with quenched staggered quarks[15].

In this article, we will compute the chiral condensate of lattice QCD with dynamical staggered quarks, using the p.d.f. method. In Sec. 2, we review the basic ideas. In Sec. 3, we present the numerical results. Some discussions are made in Sec. 4.

\section{FORMULATION}

Let us characterize each vacuum state by $\alpha$ and the chiral condensate by $\langle\bar{\psi} \psi\rangle_{\alpha}$. The p.d.f. of the chiral condensate in the Gibbs state is defined by $[11,15]$

$$
P(c)=\sum_{\alpha} w_{\alpha} \delta\left(c-\langle\bar{\psi} \psi\rangle_{\alpha}\right)
$$


with $w_{\alpha}$ the weight to get the vacuum state $\alpha . P(c)$ tells us the probability to get the value $c$ for the chiral condensate from a randomly chosen vacuum state. If the ground state is invariant under the global chiral transformation, then $P(c)=\delta(c)$. If the ground state breaks the chiral symmetry, $P(c)$ will be a more complex function, depending on the group of global chiral symmetry. Therefore, from the shape of the function $P(c)$ computed in the configurations generated by a chiral symmetric action with exact $m=0$, one can qualitatively judge whether chiral symmetry is spontaneously broken.

In quantum field theory with fermions, chiral-symmetry breaking is dominated by the properties of the fermion fields under global chiral transformation. From Eq. (4), one can define [15] the p.d.f. of the chiral condensate for a single gauge configuration $U$ :

$$
P_{U}(c)=\frac{\int[d \bar{\psi}][d \psi] \exp \left(-S_{f}\right) \delta\left[c-\frac{\sum_{x} \bar{\psi}(x) \psi(x)}{N_{c} V}\right]}{\int[d \bar{\psi}][d \psi] \exp \left(-S_{f}\right)}
$$

where in " $\sum_{x} \bar{\psi}(x) \psi(x)$ ", summation over color, spin and position indices is implied.

For gauge theory with fermions, if the action is invariant under $U(1)$ chiral transformation, we show [15] from p.d.f. that one can use

$$
C(j)=\left\langle c_{0}(U)\right\rangle=\frac{z(j)}{N_{c} V}\left\langle\frac{1}{\lambda_{j}}\right\rangle
$$

to compute the chiral condensate in the exact chiral limit. $c_{0}(U)$ is the amplitude of the chiral condensate for configuration $U$ in the $m=0$ case. $z(j)$ is the $j$-th zero of $J_{0}$, with $J_{0}$ the zeroth order Bessel function of the first kind. The average is taken over gauge configurations with dynamical fermions at $m=0$. In the chiral-symmetry breaking phase, a plateau for $C(j)=$ const. will develop, from which the chiral condensate in the chiral limit can be extracted.

The relation between eigenmodes and chiral-symmetry breaking is clear: if chiral symmetry is spontaneously broken, i.e., $c_{0}(U) \not \equiv 0$, according to Eq. (6), $\lambda_{j}$ should scale as $z(j) / V$. In the infinite volume limit $V \rightarrow \infty$, the eigenvalues relevant for chiral-symmetry breaking are those going to zero as $1 / V$, which is consistent with Banks-Casher.

The advantage of Eq. (6) is that to extract the value of $C(j)$ from a plateau, only a few smallest eigenvalues for configurations with dynamical quarks at $m=0$ are needed for this calculation. Of course, finite size analysis $\lim _{V \rightarrow \infty} C(j)$ remains to be done, as in all approaches.

\section{RESULTS FOR QCD WITH DYNAMICAL QUARKS}

In Ref. [15], we have displayed how the method works in quenched lattice QCD. Here we would like to present the first data for SU(3) LGT with dynamical staggered (Kogut-Susskind) quarks. The action is $S=S_{g}+S_{f}$ :

$$
\begin{aligned}
S_{g} & =-\frac{\beta}{N_{c}} \sum_{p} \operatorname{Re} \operatorname{Tr}\left(U_{p}\right), \\
S_{f} & =\sum_{x, y} \bar{\psi}(x) \Delta_{x, y} \psi(y),
\end{aligned}
$$




$$
\begin{aligned}
U_{p} & =U_{\mu}(x) U_{\nu}(x+\mu) U_{\mu}^{\dagger}(x+\nu) U_{\nu}^{\dagger}(x), \\
\Delta_{x, y} & =m \delta_{x, y}+\sum_{\mu=1}^{4} \frac{1}{2} \eta_{\mu}(x)\left[U_{\mu}(x) \delta_{x, y-\hat{\mu}}-U_{\mu}^{\dagger}(x-\hat{\mu}) \delta_{x, y+\hat{\mu}}\right], \\
\eta_{\mu}(x) & =(-1)^{x_{1}+x_{2}+\ldots+x_{\mu-1}},
\end{aligned}
$$

where $\beta=2 N_{c} / g^{2}$, with $N_{c}=3$. In the chiral limit $m=0$, there exists a U(1) subgroup of the continuous chiral symmetry in the fermionic action and Eq. (6) applies. All simulations are done on the $V=10^{4}$ lattice using the method described in [16].

Figure 1 shows the data for $\langle\bar{\psi} \psi\rangle$ for flavor number $N_{f}=1$ at $\beta=5.1069$ obtained from Eq. (3) at 14 nonzero $m$. For conventional simulation methods, it is very expensive to do computations at so many quark masses. A linear function is also used to extrapolate the data of $\langle\bar{\psi} \psi\rangle$ at $m \neq 0$ to the chiral limit. At this coupling, the linear fit works well for wide range of $m \in[0.004,0.1]$, so that the result can be used for comparison.

Figure 2 shows the result of $C(j)$ for the same $\beta$ and $N_{f}$ using Eq. (6) and from only one simulation[16] at $m=0$. There is a nice plateau for $j \in[5,40]$. The result is consistent with that from Eq. (3) extrapolated to the chiral limit and Eq. (6).

Figures 3-8 show respectively the results for $N_{f}=2$ at $\beta=5.0238, N_{f}=3$ at $\beta=4.9411$, and $N_{f}=4$ at $\beta=4.8590$. In the chiral limit $m=0$, the data for different flavors have the same plaquette energy $E_{p}=1.2921$, but different $\beta$, due to the effects of dynamical quarks. Our results indicate that the p.d.f. method gives estimate for the chiral condensate, consistent with the conventional one.

\section{DISCUSSIONS}

To summarize, we have extended the p.d.f. method to lattice QCD with dynamical staggered quarks for computing the chiral condensate in the exact chiral limit. The first results are very encouraging. This might be an alternative efficient method for obtaining quantitative information on the spontaneous chiral-symmetry breaking in QCD. There are several advantages: only calculations of a small set of eigenvalues of the massless Dirac operator are necessary; only one simulation at $m=0$ has to been done at one $\beta$; there is no need for $m$ or $\lambda$ extrapolation.

In some cases, i.e., when the system is at the criticality, fitting the data obtained from obtained from Eq. (3) with some modeled function might give arbitary results, while using Eq. (6) might hopefully give more reliable estimate.

\section{Acknowledgments}

I am grateful to V. Azcoiti and V. Laliena for useful discussions.

\section{References}

[1] K. G. Wilson, Phys. Rev. D 10, 2445 (1974). 
[2] S. Muroya, A. Nakamura, C. Nonaka and T. Takaishi, Prog. Theor. Phys. 110, 615 (2003), and refs. therein.

[3] S. D. Katz, Nucl. Phys. B (Proc. Suppl.) 129, 60 (2004), and refs. therein.

[4] M. P. Lombardo, hep-lat/0401021, and refs. therein.

[5] X. Q. Luo, Phys. Rev. D 70, 091504 (Rapid Commun.) (2004).

[6] E. B. Gregory, S. H. Guo, H. Kröger and X. Q. Luo, Phys. Rev. D 62, 054508 (2000).

[7] X. Q. Luo, E. B. Gregory, S. H. Guo and H. Kröger, hep-ph/0011120.

[8] Y. Fang and X. Q. Luo, Phys. Rev. D 69, 114501 (2004).

[9] X. Q. Luo and H. S. Chen, Nucl. Phys. B(Proc. Suppl.) 140, 511 (2005).

[10] H. S. Chen and X. Q. Luo, Phys. Rev. D 72, 034504 (2005).

[11] V. Azcoiti, V. Laliena and X.Q. Luo, Phys. Lett. B 354, 111 (1995).

[12] V. Azcoiti and A. Galante, Phys. Rev. Lett. 83, 1518 (1999).

[13] V. Azcoiti, G. Di Carlo, A. Galante and V. Laliena, Phys. Rev. Lett. 89, 141601 (2002).

[14] R. Aloisio, V. Azcoiti, G. Di Carlo, A. Galante and A. F. Grillo, Nucl. Phys. B 606, 322 (2001).

[15] X. Q. Luo, Phys. Rev. D 69, 076012 (2004).

[16] X. Q. Luo, Mod. Phys. Lett. A 16, 1615 (2001). 


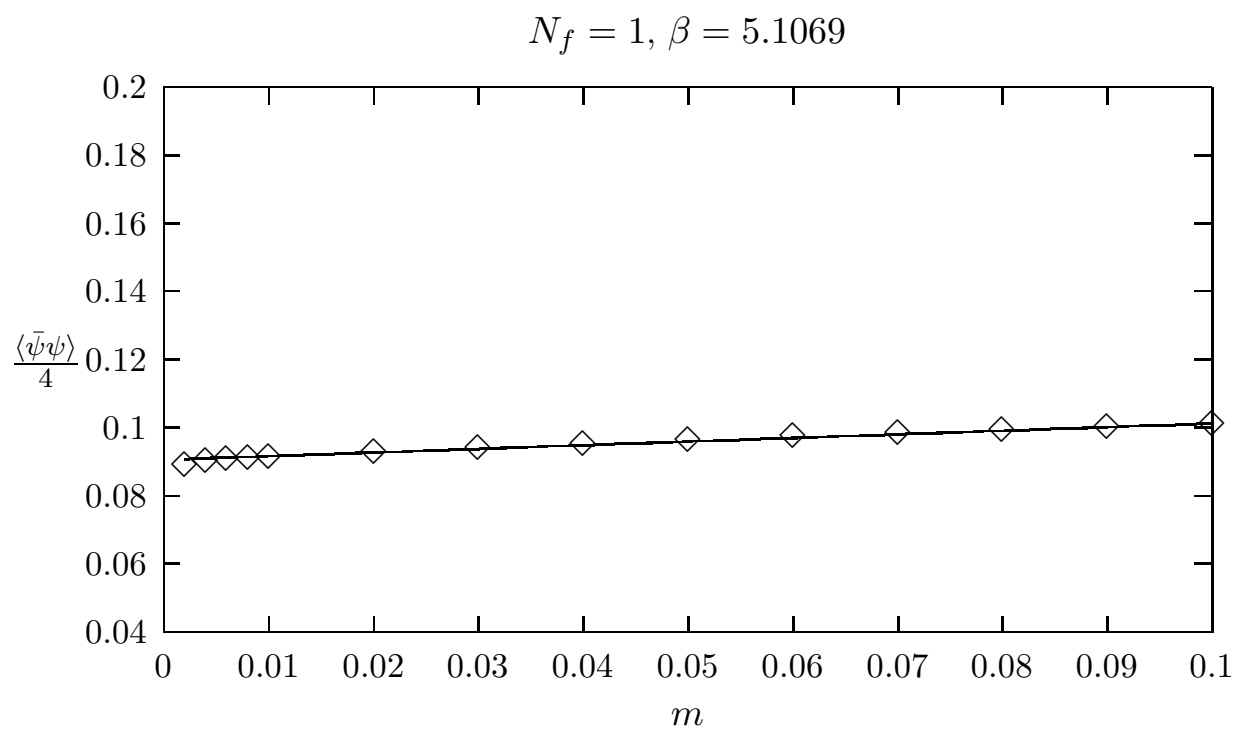

Figure 1: $\langle\bar{\psi} \psi\rangle / 4$ as a function of $m$ for $N_{f}=1$ and $\beta=5.1069$ using Eq. (3).

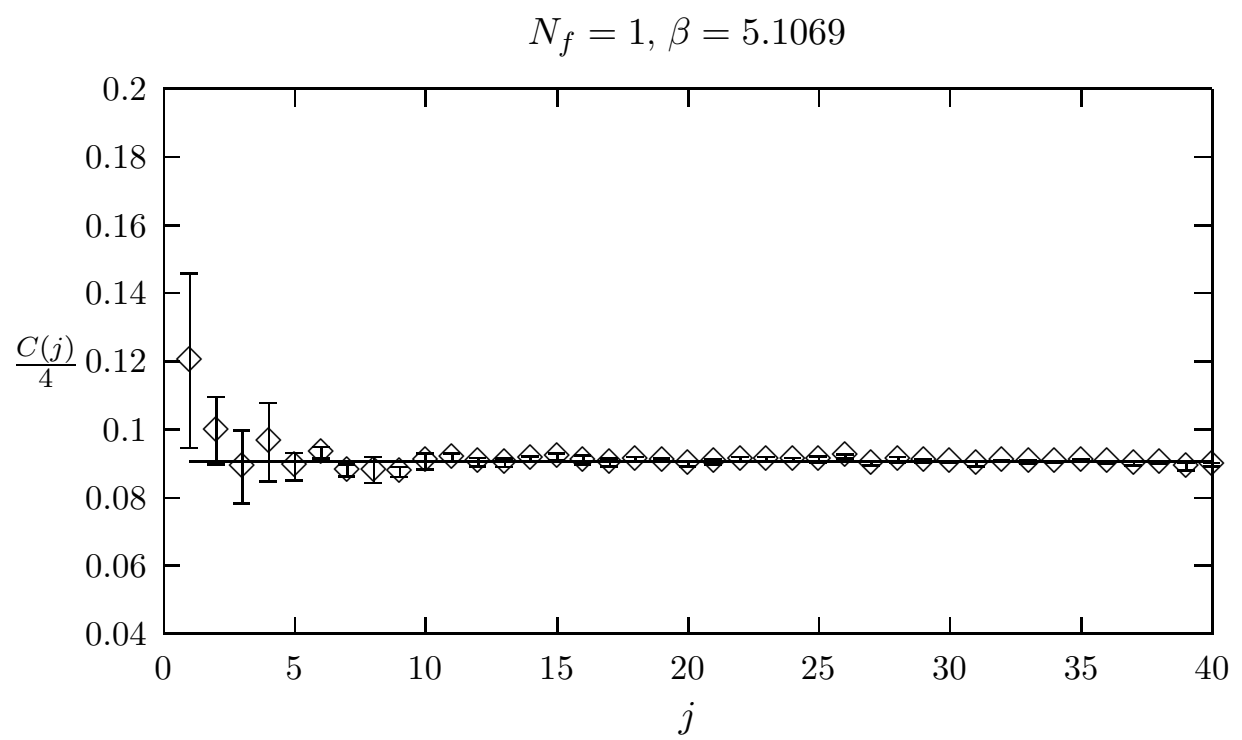

Figure 2: $C(j) / 4$ as a function of $j$ for $N_{f}=1$ and $\beta=5.1069$ using Eq. (6). 


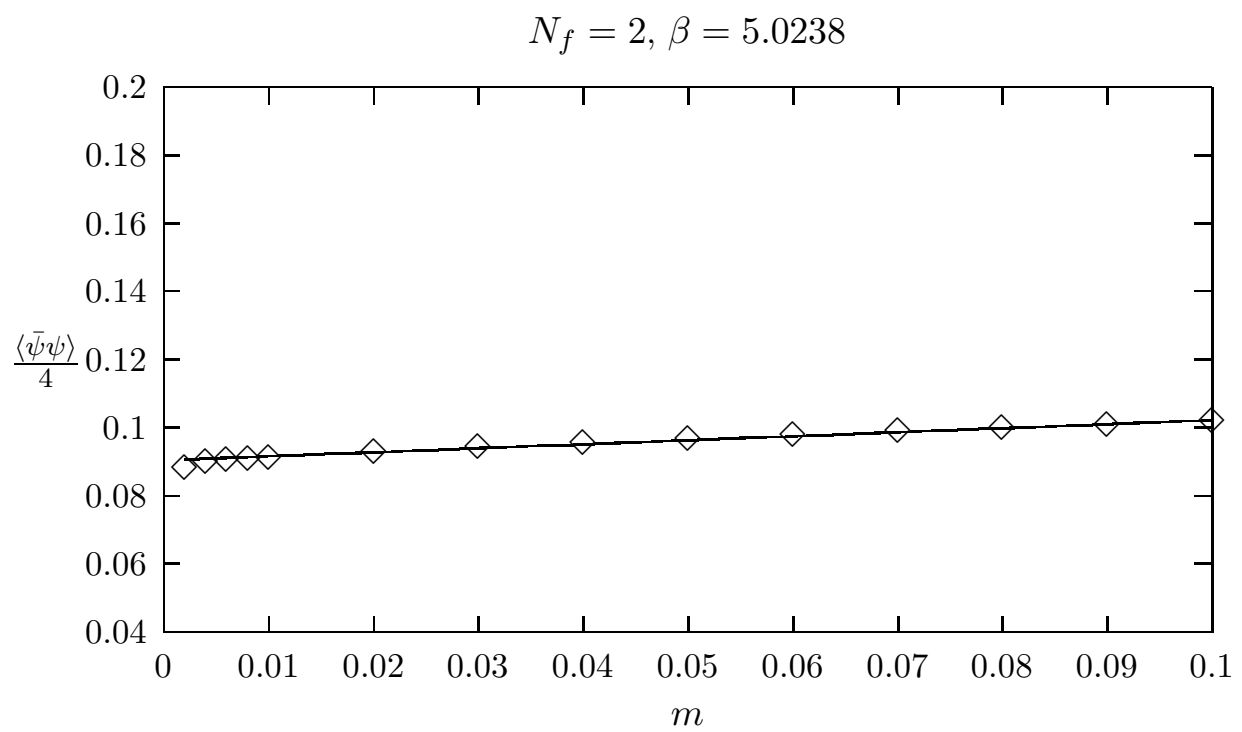

Figure 3: $\langle\bar{\psi} \psi\rangle / 4$ as a function of $m$ for $N_{f}=2$ and $\beta=5.0238$ using Eq. (3).

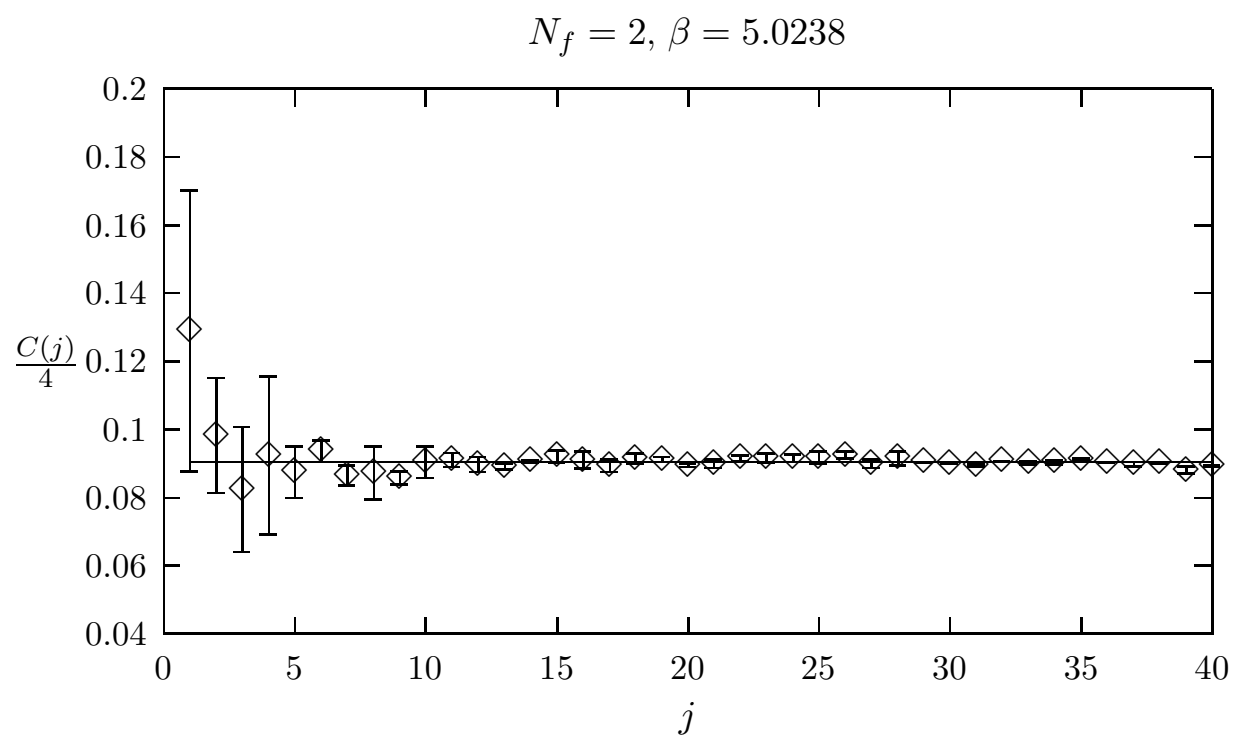

Figure 4: $C(j) / 4$ as a function of $j$ for $N_{f}=2$ and $\beta=5.0238$ using Eq. (6). 


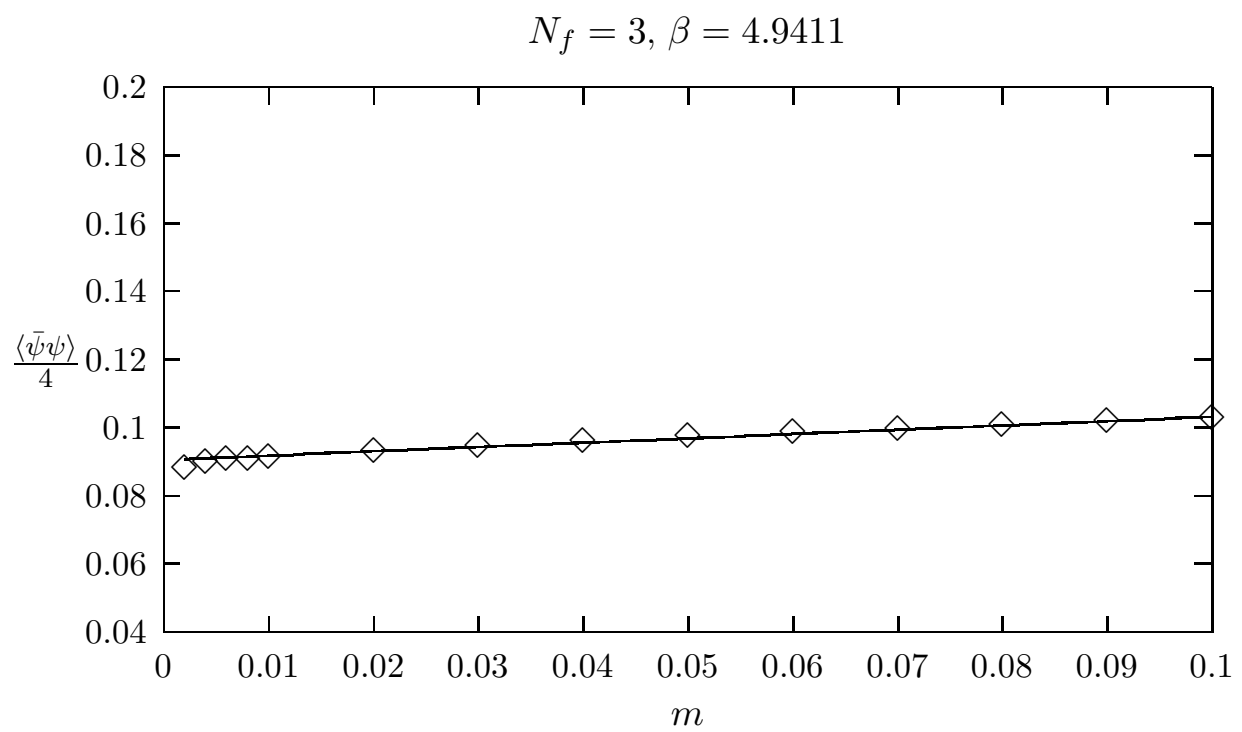

Figure 5: $\langle\bar{\psi} \psi\rangle / 4$ as a function of $m$ for $N_{f}=3$ and $\beta=4.9411$ using Eq. (3).

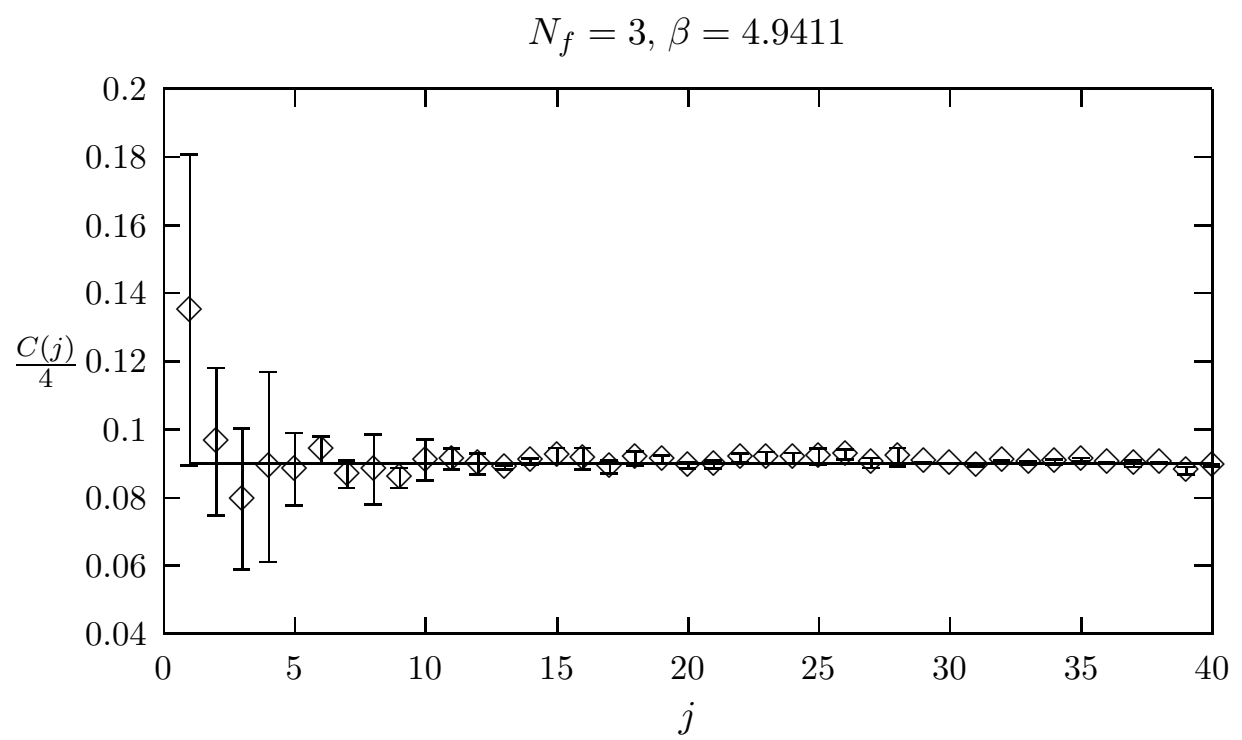

Figure 6: $C(j) / 4$ as a function of $j$ for $N_{f}=3$ and $\beta=4.9411$ using Eq. (6). 


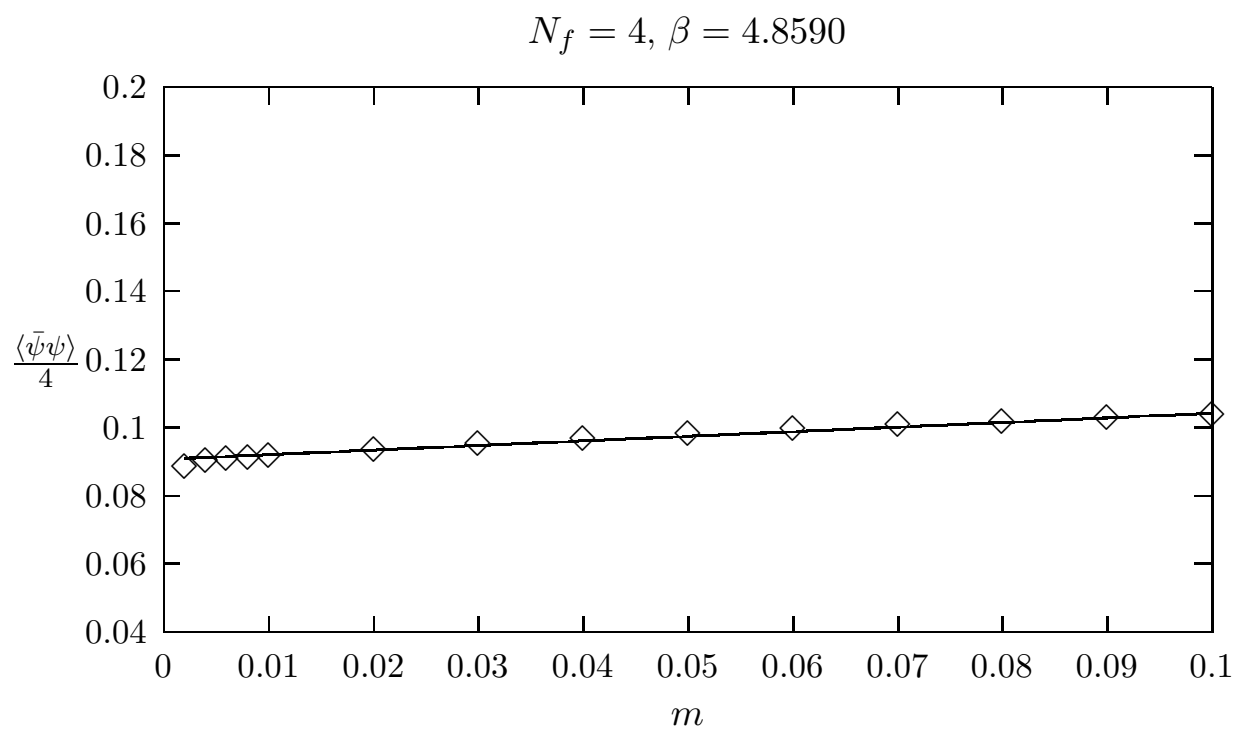

Figure 7: $\langle\bar{\psi} \psi\rangle / 4$ as a function of $m$ for $N_{f}=4$ and $\beta=4.8590$ using Eq. (3).

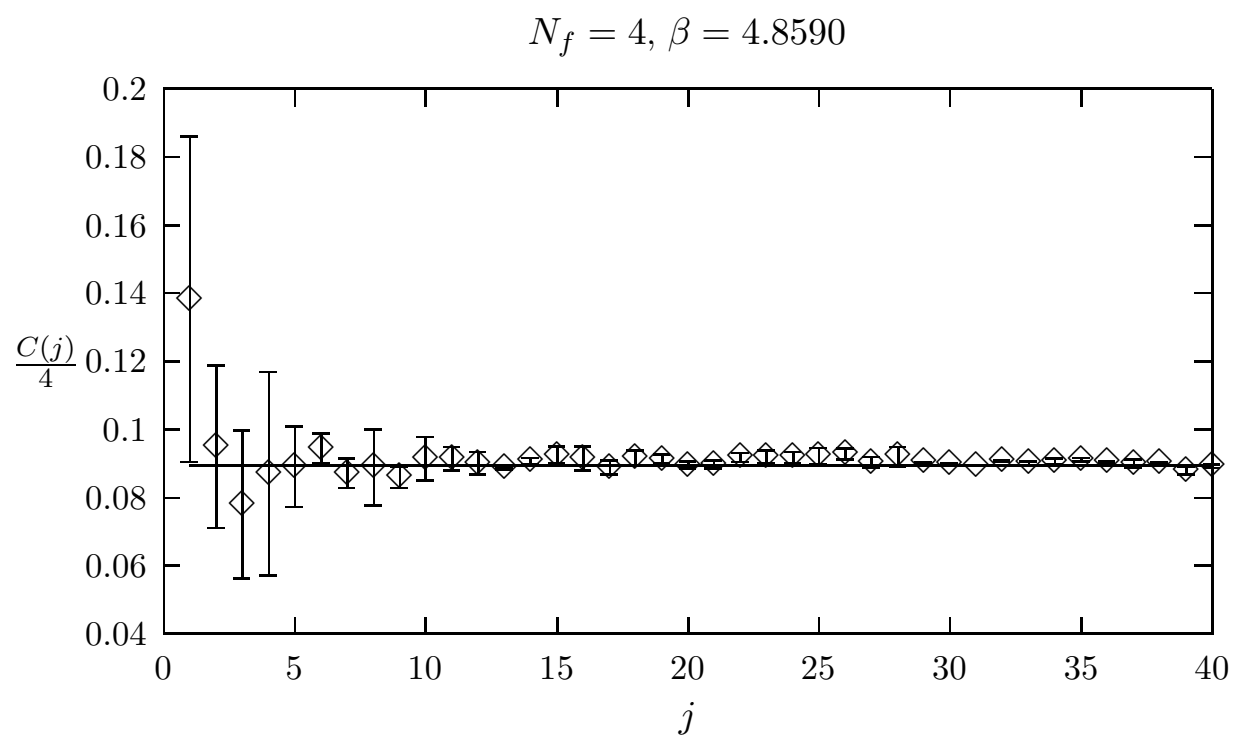

Figure 8: $C(j) / 4$ as a function of $j$ for $N_{f}=4$ and $\beta=4.8590$ using Eq. (6). 